\title{
Forward-thinking design solutions for mechanical circulatory support: multifunctional hybrid continuous-flow ventricular assist device technology
}

\author{
Amy Throckmorton ${ }^{1}$, Ellen Garven ${ }^{1}$, Matthew Hirschhorn ${ }^{1}$, Steven Day ${ }^{2}$, Randy Stevens ${ }^{3,4}$, \\ Vakhtang Tchantchaleishvili ${ }^{5}$ \\ ${ }^{1}$ BioCirc Research Laboratory, School of Biomedical Engineering, Science, and Health Systems, Drexel University, Philadelphia, PA USA; \\ ${ }^{2}$ Department of Biomedical Engineering, Kate Gleason College of Engineering, Rochester Institute of Technology, Rochester, NY, USA; ${ }^{3}$ Pediatrics, \\ College of Medicine, Drexel University, Philadelphia, PA, USA; ${ }^{4}$ Heart Center for Children, St. Christopher's Hospital for Children, Philadelphia, \\ PA, USA; ${ }^{5}$ Division of Cardiac Surgery, Department of Surgery, Thomas Jefferson University Hospital, Philadelphia, PA, USA \\ Correspondence to: Amy Throckmorton, PhD, Associate Professor. School of Biomedical Engineering, Science and Health Systems, Drexel University, \\ 3141 Chestnut Street, Rm. 718, Philadelphia, PA 19104, USA. Email: Amy.Throckmorton@drexel.edu.
}

Submitted Sep 16, 2020. Accepted for publication Nov 18, 2020.

doi: 10.21037/acs-2020-cfmcs-14

View this article at: http://dx.doi.org/10.21037/acs-2020-cfmcs-14

The field of mechanical circulatory support (MCS) has benefited from advances that have facilitated a paradigm shift from pulsatile blood pumps to continuous flow (CF) pumps (1). These CF pumps offer significant design advantages over pulsatile, positive displacement devices. Compared to CF technology, pulsatile pumps experience more frequent mechanical failures due to failing valves and membranes from repetitive flexions. Recent advances in CF-ventricular assist devices (VADs) involve design innovation of their power systems, such as the use of magnetic bearings in lieu of mechanical or fluid film bearings. VADs in clinical use and under various stages of development, however, have several ongoing clinical limitations and challenges, including: thromboembolic events, neurologic impairment, risk of infection and the lack of smaller options for pediatric patients $(1,2)$. Specifically, for children, there is no VAD technology that can satisfy their distinct design requirements: wide range of patient sizes, increased cardiovascular demand with growth, and anatomic and physiologic heterogeneity of congenital heart disease (3). Despite improvement, these critical limitations of blood pump designs persist and create pathway-limiting barriers to sustained clinical progress. Few design advances in blade geometry or pump components have occurred to address these unmet challenges. Here we highlight several forward-thinking design solutions: pitch-adjusting blades; hybrid pump configurations; design simplicity; dual-support features; weight, wearables and wireless power systems; switching operational capabilities; excellent hemocompatibility; and versatility in therapy.

\section{Pitch-adjusting blades}

Virtually all CF-VADs have fixed-bladed impeller designs that produce superior flow conditions at a particular design operating point, and it is known that there diminished flow control when the blood pump is operated at off-design conditions. The cardiovascular system is dynamic and timevarying, thus flow conditions through VADs are off-design during the majority of the cardiac cycle. This inherently increases the risk of thrombosis and hemolysis. There has been limited investigation of controlled pitch-adjustment blade characteristics as a means of flow control and enhanced energy transfer. The concept of pitch-adjusting blades is defined as controlled geometric blade twist or angle changes such that blood flow striking the leading blade edge has reduced resistance and efficient alignment, producing maximal pressure or thrust (4). Controlled or passive blade angle adjustment could modulate the pressure generation of a blood pump without the need to increase rotational speed. Thus, it is plausible to dynamically change the impeller blade angles during MCS to achieve patientspecific or disease-specific pressure and capacity operational requirements. 


\section{Hybrid pump configurations}

CF-VAD technology generally includes a single axial or centrifugal blood pump. Hybrid designs are now being realized whereby axial and centrifugal pumps are uniquely integrated within a single pump housing. A centrifugal impeller, for example, could rotate around a separate axial pump domain, with both impellers rotating around a common central axis (5). This design integrates two blood pumps into the housing space typically afforded to one VAD, allowing this device to be more compact and effectively support high-risk patients.

\section{Design simplicity}

Design innovation will continue to advance this technology and to reduce design complexity. The design of novel combined, motor-magnetic suspension drive systems will facilitate unobstructed blood flow paths, longer operational lifespans (fifteen years), lower component wear, as well as wider clearances between rotating and stationary surfaces, reducing fluid stresses and the risk of thrombosis and hemolysis $(6,7)$.

\section{Dual-support features}

Recent studies suggest that there are benefits to flow pulsatility in chronic MCS to alleviate bleeding that may develop in the digestive track due to cleavage of von-Willebrand factor (8). Thus, the field has pivoted, implementing and developing new control algorithms that modulate the rotational speed of VADs to produce pulsatile pressure and blood flow waveforms.

\section{Weight, wearables \& wireless power systems}

The use of composite materials in VADs will ensure that the drive consoles and wearable components (i.e., controller, dual-battery pack, support holster) are lighter, safer and more portable options. Moreover, wearable electronic devices with micro-controllers could be integrated into VAD materials to detect, analyze and transmit biosignals, vital signs and activity tracking data. This data could serve as biofeedback to inform physiologic control and thus, automatically regulate VAD operational states. To address abdominal driveline and power consumption challenges, the implementation of novel transcutaneous energy transfer (TET) power systems based on wireless technology, coplanar inductive coupling energy systems or high efficiency resonant coupling unit using magnetic fields is also becoming realizable $(9,10)$.

\section{Switching operational capabilities}

Unique positioning of blood pumps under a single pump housing creates versatility in potential therapy. This presents the prospect to operationally switch from one pump to another. Multiple blood pumps could be aligned in a series, parallel or gyroscopic configuration under a common housing. Strategic orientation of pumps within a single device housing could provide the means to shunt blood flow from one VAD to another upon activation of a switching mechanism. In cases where one blood pump fails, the secondary pump could be activated. The means to toggle from one blood pump to another in a single device could also address the challenges of MCS for pediatric patients during growth and development, from infancy to adolescence.

\section{Excellent hemocompatibility}

Superhydrophobic surface characteristics and roughness modifications, biocoatings with drug eluting properties, and biomaterial surface layers with regenerative properties are newly emerging innovative, cross-disciplinary and synergistic design solutions to the ongoing challenges of hemocompatibility and thrombotic risks.

\section{Versatility in therapy}

Clinical teams require versatility in therapy as they consider the right treatment for the right patient at the appropriate time. The integration of two or more blood pumps into one VAD technology means that this device could operate in a multitude of ways. It could be employed as only a right ventricular assist device (RVAD), only a left ventricular assist device (LVAD), a total artificial heart (TAH) for partial or full cardiovascular support and, either paracorporally or intracorporally (5). These highlighted design advances are only a few in the pipeline as we survey the landscape of emerging innovation in the field of MCS. New, forwardthinking design solutions are required as we seek to innovate novel VAD technology into the $21^{\text {st }}$ century and transform clinical treatment paradigms for all patients with congestive heart failure, secondary to acquired or congenital heart disease. As a field, we continue to build on the rich and inspirational history of yesterday's innovation 
as we doggedly pursue the translational development of tomorrow's new life-saving solutions.

\section{Acknowledgments}

Funding: None.

\section{Footnote}

Conflicts of Interest: The authors have no conflicts of interest to declare.

Open Access Statement: This is an Open Access article distributed in accordance with the Creative Commons Attribution-NonCommercial-NoDerivs 4.0 International License (CC BY-NC-ND 4.0), which permits the noncommercial replication and distribution of the article with the strict proviso that no changes or edits are made and the original work is properly cited (including links to both the formal publication through the relevant DOI and the license). See: https://creativecommons.org/licenses/by-nc-nd/4.0/.

\section{References}

1. Olsen DB. The history of continuous-flow blood pumps. Artif Organs 2000;24:401-4.

2. Fox CS, McKenna KL, Allaire PE, et al. Total Artificial Hearts-Past, Current, and Future. J Card Surg

Cite this article as: Throckmorton A, Garven E, Hirschhorn M, Day S, Stevens R, Tchantchaleishvili V. Forward-thinking design solutions for mechanical circulatory support: multifunctional hybrid continuous-flow ventricular assist device technology. Ann Cardiothorac Surg 2021;10(3):383385. doi: 10.21037/acs-2020-cfmcs-14
2015;30:856-64.

3. Chopski SG, Moskowitz WB, Stevens RM, et al. Mechanical circulatory support devices for pediatric patients with congenital heart disease. Artif Organs 2017;41:E1-E14.

4. Throckmorton AL, Sciolino MG, Downs EA, et al. Controlled pitch-adjustment of impeller blades for an intravascular blood pump. ASAIO J 2012;58:382-9.

5. Fox C, Chopski S, Murad N, et al. Hybrid continuousflow total artificial heart. Artif Organs.2018;42:500-9.

6. Day SW, Cheng S, Olles MW, et al. Development of a single path magnetically levitated axial flow blood pump. Artificial Organs 2010;34:E154.

7. Cheng S, Olles MW, Olsen DB, et al. Miniaturization of a magnetically levitated axial flow blood pump. Artif Organs 2010;34:807-15.

8. Coghill PA, Kanchi S, Azartash-Namin ZJ, et al. Benchtop von Willebrand Factor Testing: Comparison of Commercially Available Ventricular Assist Devices and Evaluation of Variables for a Standardized Test Method. ASAIO J 2019;65:481-8.

9. Bocan KN, Sejdić E. Adaptive Transcutaneous power transfer to implantable devices: a state of the art review. Sensors (Basel) 2016;16:393.

10. Kassif Y, Zilbershlag M, Levi M, et al. A New Universal Wireless Transcutaneous Energy Transfer (TET) System for Implantable LVADs - Preliminary In Vitro and In Vivo Results. J Heart Lung Transplant 2013;32:S140-1. 